\title{
Vertically coupled quantum dots in the local spin-density functional theory
}

\author{
Martí Pi, Agustí Emperador, and Manuel Barranco \\ Department d'Estructura i Constituents de la Matèria, Facultat de Física, Universitat de Barcelona, E-08028 Barcelona, Spain
}

Francesca Garcias

Department de Física, Facultat de Ciències, Universitat de les Illes Balears, E-07071 Palma de Mallorca, Spain

(Received 27 April 2000; revised manuscript received 27 November 2000; published 1 March 2001)

\begin{abstract}
We have investigated the structure of double quantum dots vertically coupled at zero magnetic field within local-spin-density functional theory. The dots are identical and have a finite width, and the whole system is axially symmetric. We first discuss the effect of thickness on the addition spectrum of one single dot. Next we describe the structure of coupled dots as a function of the interdot distance for different electron numbers. Addition spectra, Hund's rule, and molecular-type configurations are discussed. It is shown that self-interaction corrections to the density-functional results do not play a very important role in the calculated addition spectra.
\end{abstract}

DOI: $10.1103 /$ PhysRevB.63.115316

PACS number(s): 73.21.-b, 71.15.Mb, 85.35.Be, 36.40.Ei

\section{INTRODUCTION}

The study of systems with a small number $(N)$ of electrons confined to a quasi-two-dimensional (2D) semiconductor quantum dot (QD) constitutes a subject of growing interest (see, for instance, Refs. 1 and 2, and references therein). One reason for this interest is that their electronic properties can be selected with some freedom by tailoring the shape of the lateral confining potential. In this sense, they are often referred to as artificial atoms. Recently, circular and elliptically disk-shaped QD's have been built in a very clean way, and their properties have been thoroughly studied ${ }^{3-9}$ as a function of $N$.

Usually, QD's are described as true 2D systems. This seems justified, as it has been widely tested by comparing theory with experiment. However, this comparison may be obscured by the fact that the parameters defining the confining potential are adjusted and might mask 3D effects. However, complex 2D calculations have been carried out without further restrictions or imposed symmetries. ${ }^{8-12}$ Full 3D calculations also exist in Hartree or Hartree-Fock approximations for very few electrons (see for instance Refs. 13 and 14), and also within the density-functional theory. ${ }^{15-18}$

A systematic study of the role of dimensionality in QD structure was presented by Rontani et al. ${ }^{19,20}$ In spite of the success of 2D models in describing the properties of QD's subjected to perpendicular magnetic fields, the width in the growth direction of most experimentally studied QD's is around one order of magnitude smaller than their typical radius. The effect of a small but finite vertical extension on the QD structure is worth studying, considering that a recent extension of single QD studies to vertically coupled quantum $\operatorname{dots}^{21}$ may render including the $z$ extension of the constituent dots unavoidable when describing the experimental data. ${ }^{4,5}$

Vertically coupled dots, also called artificial or quantum dot molecules, were theoretically addressed in a number of works. ${ }^{22-27}$ Only in Ref. 22 was the $z$ extension of the constituent QD's taken into account; in the other references, it was neglected, and consequently their results cannot be reliable when the interdot distance is comparable to their $z$ extension, which is an interesting physical situation. ${ }^{4,5}$
In this work we present a study of vertically coupled, cylindrical QD's in the local-spin-density-functional theory (LSDFT). We restrict our description to axially symmetric configurations and identical QD's, and limit ourselves to a zero magnetic field $B$. While these two latter conditions are easy to relax without increasing the amount of numerical work too much, axial symmetry breaking would require a more demanding 3D calculation.

Our scheme is based on the application of the so-called ${ }^{28}$ imaginary-time method (ITM). It requires a discretization of the Kohn-Sham (KS) equations on a spatial mesh that can be straightforwardly implemented on a personal computer, and avoids expansion of the single-particle (sp) wave functions in a basis, large matrix diagonalizations and tests of the stability of the results against changes in the size of the basis.

In contrast to standard methods for computing molecular structure ${ }^{29}$ we do not postulate from the start that artificial molecular orbitals can be expressed as linear combinations of artificial atomic orbitals, and consequently we avoid making approximations such as complete neglect of differential overlap where the individual wave functions of electrons associated with different artificial atoms are taken as being orthonormal, as happens when the QD's are either two dimensional or far apart.

The present approach to the description of vertically coupled QD's constitutes a clear improvement on previous LSDFT calculations, ${ }^{27}$ in which the electrons are located on one of the dots, and as a consequence, can only be electrostatically coupled, even when they lie at short distances. With respect to the generalized Hubbard model plus the diagonalization scheme of Ref. 22, the present improvement is the possible application of the LSDFT to systems with large numbers of electrons. Moreover, one could think of a trivial generalization to non-identical dots, paying the well known token that the LSDFT treats the exchange Coulomb term locally, and that LSDFT configurations are usually mixtures of many-electron states with the same value of the total spin projection $S_{z}$, though with different total spin $S$. For small systems, this drawback (which is also inherent to the generalized Hubbard approach ${ }^{22}$ ) can be removed with a shellmodel calculation in a restricted sp valence space. ${ }^{30,31} \mathrm{An}$ - 
other difference between our approach and the Hubbard model is that even if the exchange energy is treated locally, it is not restricted to involving only electrons located on the same dot. This might be advantageous in the strong-coupling case, when the dots are close together and the quantummechanical coupling due to electron exchange is important.

This paper is organized as follows. In Sec. II we present the formalism and the essentials of the ITM. Results for one single thick QD and for two coupled dots are presented in Sec. III, and a summary is presented in Sec. IV. In the Appendix we present the self-interaction correction $^{32}$ to the density-functional results obtained for two extreme interdot distances and several electron numbers.

\section{METHODOLOGICAL APPROACH}

Within the LSDFT, the ground state of the system is obtained by solving the Kohn-Sham equations. The problem is simplified by the imposed axial symmetry around the $z$ axis, which allows one to write the sp wave functions as $\phi_{n l \sigma}(r, z, \theta, \sigma)=u_{n l \sigma}(r, z) e^{-i l \theta} \chi_{\sigma} \quad$ with $\quad l=0, \pm 1, \pm 2 \ldots$, where $-l$ is the projection of the sp orbital angular momentum on the symmetry axis.

We have used effective atomic units $\hbar=e^{2} / \epsilon=m=1$, where $\epsilon$ is the dielectric constant, and $m$ the electron effective mass. In units of the bare electron mass $m_{e}$ one has $m$ $=m^{*} m_{e}$. In this system, the length unit is the effective Bohr radius $a_{0}^{*}=a_{0} \epsilon / m^{*}$, and the energy unit is the effective Hartree $H^{*}=H m^{*} / \epsilon^{2}$. In the numerical applications we have considered GaAs, for which we have taken $\epsilon=12.4$, and $m^{*}=0.067$. This yields $a_{0}^{*} \sim 97.94 \AA$ and $H^{*} \sim 11.86 \mathrm{meV}$.

In cylindrical coordinates the KS equations read

$$
\begin{aligned}
& {\left[-\frac{1}{2}\left(\frac{\partial^{2}}{\partial r^{2}}+\frac{1}{r} \frac{\partial}{\partial r}-\frac{l^{2}}{r^{2}}+\frac{\partial^{2}}{\partial z^{2}}\right)+V_{c f}(r, z)\right.} \\
& \left.+V^{H}+V^{x c}+W_{x c} \eta_{\eta}\right] u_{n l \sigma}(r, z)=\epsilon_{n l \sigma} u_{n l \sigma}(r, z),
\end{aligned}
$$

where $\eta_{\sigma}=+1(-1)$ for $\sigma=\uparrow(\downarrow), V_{c f}(r, z)$ is the confining potential, $V^{H}(r, z)$ is the direct Coulomb potential, and $V^{x c}$ $=\partial \mathcal{E}_{x c}(n, m) /\left.\partial n\right|_{g s}$ and $W^{x c}=\partial \mathcal{E}_{x c}(n, m) /\left.\partial m\right|_{g s}$ are the variations of the exchange-correlation energy density $\mathcal{E}_{x c}(n, m)$ in terms of the electron density $n(r, z)$ and of the local spin magnetization $m(r, z) \equiv n^{\uparrow}(r, z)-n^{\downarrow}(r, z)$ taken at the ground state (gs).

As usual, $\mathcal{E}_{x c}(n, m) \equiv \mathcal{E}_{x}(n, m)+\mathcal{E}_{c}(n, m)$ has been built from 3D homogeneous electron-gas calculations. This yields a well-known, ${ }^{33}$ simple analytical expression for the exchange contribution $\mathcal{E}_{x}(n, m)$. For the correlation contribution $\mathcal{E}_{c}(n, m)$ we have used two different parametrizations, both based on the results of Ceperley and Alder. ${ }^{34}$ The first was proposed by Vosko, Wilk, and Nusair, ${ }^{35}$ and the second by Perdew and Zunger. ${ }^{32}$ We have checked that they yield the same results, and all results presented in this work were obtained with the exchange-correlation energy density proposed by Perdew and Zunger.
For a double QD the confining potential $V_{c f}(r, z)$ has been taken to be parabolic with frequency $\omega_{0}$ in the $x y$ plane, plus a symmetric double quantum well in the $z$ direction. For a single QD we have also used a parabolic confining potential in the $x y$ plane, together with a quantum well in the $z$ direction. Any other axially symmetric $V_{c f}(r, z)$ can be implemented as well.

$V^{H}(r, z)$ was obtained solving the Poisson equation using the conjugate gradient method ${ }^{28,37}$ (CGM). This requires a knowledge of $V^{H}(r, z)$ at the mesh boundary, which can be obtained by direct integration. Due to axial symmetry

$$
\begin{aligned}
V^{H}(r, z)= & 2 \int_{0}^{\infty} r^{\prime} d r^{\prime} \int_{-\infty}^{+\infty} d z^{\prime} \Delta n\left(\mathbf{r}^{\prime}\right)\left[\left(r+r^{\prime}\right)^{2}\right. \\
& \left.+\left(z-z^{\prime}\right)^{2}\right]^{1 / 2} \mathbf{E}\left(\alpha^{2}\right),
\end{aligned}
$$

where $\mathbf{E}$ is the complete elliptic integral of the second kind, ${ }^{36}$ and $\alpha^{2} \equiv 4 r r^{\prime} /\left[\left(r+r^{\prime}\right)^{2}+\left(z-z^{\prime}\right)^{2}\right]$.

We have discretized the KS and Poisson equations using $k$-point Lagrange formulas for the $r$ and $z$ derivatives, and $(k+1)$-point Lagrange formulas for the integrations. ${ }^{36}$ The mesh size has to be such that the discretized wave functions $u\left(r_{i}, z_{j}\right)$ at the mesh boundary can be safely taken as zero. This is one boundary condition for physically acceptable solutions to Eq. (1), the other one is the regularity of the sp wave functions at $r=0$. In our scheme the $\Delta r$ and $\Delta z$ steps may have different values. The high precision demanded by the calculation imposes restrictions on the possible values of $k, \Delta r$, and $\Delta z$, and will be discussed below. This space discretization scheme offers an efficient calculation of sp wave functions, and thus of the electron densities and direct Coulomb and exchange-correlation potentials.

The imaginary time method is described in detail in Ref. 28. It is based on the observation that the discretized timeevolution operator $\mathcal{H}(t)$ for the time-dependent KS equations

$$
i \hbar \frac{\partial \phi_{j}(t)}{\partial t}=\mathcal{H}(t) \phi_{j}(t)
$$

which formally yields

$$
\Psi_{j}^{(n+1)}=\exp \left(-\frac{i}{\hbar} \Delta t \mathcal{H}^{(n)}\right) \phi_{j}^{(n)},
$$

where $\Delta t$ is the time step and $n$ indicates the step iteration, for imaginary $\Delta t=-i \Delta \tau(\Delta \tau>0)$ produces a decrease of the KS energy. In imaginary time, the wave functions $\left\{\Psi_{j}^{(n+1)}\right\}$ are no longer orthonormal, and a Gram-Schmidt orthonormalization has to be carried out after each iteration to obtain $\left\{\phi_{j}^{(n+1)}\right\}$ from $\left\{\Psi_{j}^{(n+1)}\right\}$. To first order in $\Delta \tau$, Eq. (4) becomes

$$
\Psi_{j}^{(n+1)}=\left(1-\frac{\mathcal{H}^{(n)} \Delta \tau}{\hbar}\right) \phi_{j}^{(n)},
$$

which shows the simplicity of the method and its practical implementation: after discretizing the KS Hamiltonian and wave functions, it essentially reduces to repeated application 
of the KS Hamiltonian to the previous wave functions. Moreover, it is easy to check from Eq. (5) that, upon convergence,

$$
\epsilon_{j}^{(n+1)}=\frac{\hbar}{\Delta \tau}\left[1-\left\langle\phi_{j}^{(n)} \mid \Psi_{j}^{(n+1)}\right\rangle\right]
$$

coincide with the KS sp energies. Equation (5) shows that the ITM belongs to the general class of relaxation methods employed to solve partial differential equations. This provides a simple criterion for fixing $\Delta \tau$ in such a way that the imaginary time evolution is stable, ${ }^{37}$ namely, $\hbar^{2} \Delta \tau_{\max } /\left(2 m \Delta^{2}\right)<1 / 4$, with $\Delta$ the smallest between the $r$ and $z$ steps. In actual calculations we have taken $\Delta \tau$ $=0.1 \Delta \tau_{\max }$.

To start the ITM iteration one needs a set of sp wave functions and energies to build the initial sp level scheme. We have used two such sets. The first consists of the wave functions of an axially symmetric, 3D harmonic oscillator potential of frequencies $\omega_{0}$ and $\omega_{z}$ in the radial and $z$ directions, respectively. This potential gives rise to analytical solutions even in the presence of a constant magnetic field in the $z$ direction, and is a suitable confining potential for a single QD. In this case, the sp Hamiltonian is separable and the wave function can be written as $u(r, z)=\mathcal{R}(r) \mathcal{Z} / \sqrt{2 \pi}$, with

$$
\begin{gathered}
\mathcal{R}(r)=\frac{1}{a} \sqrt{\frac{n_{r} !}{2^{|l|}\left(n_{r}+|l|\right) !}}\left(\frac{r}{a}\right)^{|l|} e^{-(r / 2 a)^{2}} L_{n_{r}}^{|l|}\left(\frac{r^{2}}{2 a^{2}}\right), \\
\mathcal{Z}(z)=\sqrt{\frac{\zeta}{\sqrt{\pi} 2^{n_{z} n_{z} !}}} H_{n_{z}}(\zeta z) e^{-\zeta^{2} z^{2} / 2},
\end{gathered}
$$

where $a \equiv \sqrt{\hbar / 2 m \omega_{0}}, \zeta \equiv \sqrt{m \omega_{z} / \hbar}$, and $L_{n_{r}}^{|l|}$ and $H_{n_{z}}$ are generalized Laguerre and Hermite polynomials, ${ }^{36}$ respectively. The sp energies are $\mathcal{E}_{n_{r}, n_{z}, l}=\hbar \omega_{0}\left[2 n_{r}+|l|+1\right]+\hbar \omega_{z}\left[n_{z}\right.$ $+1 / 2]$, with $n_{r}$ and $n_{z}$ equal to $0,1, \ldots$.

For a double QD, we have found it convenient to choose $\mathcal{Z}(z)$ as the lowest energy eigenfunctions of a 1D double quantum well. The QD thickness is such that for not too many electrons, only the two lowest states are needed. If the double dot is symmetric, these solutions are either even or odd under reflection $z \rightarrow-z$. Actually, for one QD, $\mathcal{Z}(z)$ can also be the wave function in the 1D quantum well.

Both for a single QD and for a symmetric double dot, the single-electron wave functions are characterized by the values of $l_{z}, s_{z}$, and parity, i.e., they must be either symmetric or antisymmetric under inversion $\mathbf{r} \rightarrow-\mathbf{r}$, and be either even or odd when reflecting $z \rightarrow-z$. All these symmetries are included in the starting separable wave functions. Indeed, the Hermite polynomials are even or odd depending on $n_{z}$, and the parity of a sp level is simply $(-)^{n_{z}+|l|}$. As the KS Hamiltonian and the ITM preserve these symmetries, in the course of the iteration procedure the sp wave functions, which are no longer separable, keep their initial quantum numbers (orbital and spin projection on the $z$ axis, parity, and reflection symmetry with respect to the $z=0$ plane) which are conserved quantities.
To check the numerical scheme we have carried out extensive and systematic tests. A first test on the discretization and iteration procedure consisted in numerically solving an axially deformed harmonic oscillator potential. We have exactly reproduced the spectrum $\mathcal{E}_{n_{r}, n_{z}, l}$ given after Eqs. (7). The implementation of the CGM has been successfully tested comparing results computed for a spherical Gaussian charge distribution with the analytical results.

As another test of the numerical code, we have compared the total energy calculated from a straightforward integration of the energy density with the expression in terms of the $\mathrm{sp}$ energies derived from the KS equations. Writing the correlation energy density ${ }^{32,35}$ as $\mathcal{E}_{c}[n, m] \equiv n \mathcal{E}_{\text {cor }}[n, \xi]$, where $\mathcal{E}_{\text {cor }}[n, \xi]$ is the correlation energy per electron and $\xi \equiv m / n$ is the local-spin polarization, one obtains

$$
\begin{aligned}
E= & \sum_{j} \epsilon_{j}-\int d \mathbf{r}\left\{\frac{1}{2} V^{H}(\mathbf{r}) n(\mathbf{r})+\frac{1}{3} \mathcal{E}_{x}[n, m]\right. \\
& \left.+\frac{\partial \mathcal{E}_{c o r}[n, \xi]}{\partial n} n^{2}(\mathbf{r})\right\} .
\end{aligned}
$$

We have checked that in the worst case, the energies calculated with either method agree to within one part in $10^{4}$ for our combination of $k=7$ formulas and the values $\Delta r=\Delta z$ $=0.12 a_{0}^{*}$ used in this work. ${ }^{38}$ Simpler three-point formulas $(k=3)$ turned out to be inaccurate for reasonable spatial steps, and $k=9$ and 11 formulas, which allow larger steps, did not appreciably reduce the computing time. It might be interesting to remark that most of this disagreement arises from the integral of the external confining potential for a quantum well in the $z$ direction, due to the sharp discontinuity in the given potential. We have checked that for a parabolic confining potential in the $z$ direction, both methods of evaluating the total energy agree to within one part in $10^{7}$.

In the case of double QD's, we have also checked that the results coincide irrespective of whether we start the iteration from pure harmonic oscillator wave functions, or from the better choice of double quantum well eigenfunctions in the $z$ direction. For even- $N$ systems we have always started the iteration with nonidentical sp potentials for spin-up and -down electrons, to avoid artificial configurations with $S_{z}$ $=0$.

\section{RESULTS}

\section{A. Single quantum dot}

We have first addressed the addition energies of one quantum dot hosting up to $N=21$ electrons. The confining potential in the $z$ direction is a quantum well $W=12 \mathrm{~nm}$ wide, which corresponds to the experimental well, ${ }^{4}$ and $V_{0}$ $=200 \mathrm{meV}$ deep ${ }^{19}$ Electrons are laterally confined by the parabolic potential $m \omega_{0}^{2} r^{2} / 2$ for which we have tried different $\omega_{0}$ values.

Figure 1 shows the addition spectrum $\Delta A(N) \equiv E(N$ $+1)-2 E(N)+E(N-1)$ for $\omega_{0}=3,5$, and $10 \mathrm{meV}$. In addition to local maxima at shell filling values $N=2,6,12$, and 20, other peaks appear at half-filling values $N=4$ and 9 . This is a consequence of Hund's rule, which establishes that degen- 


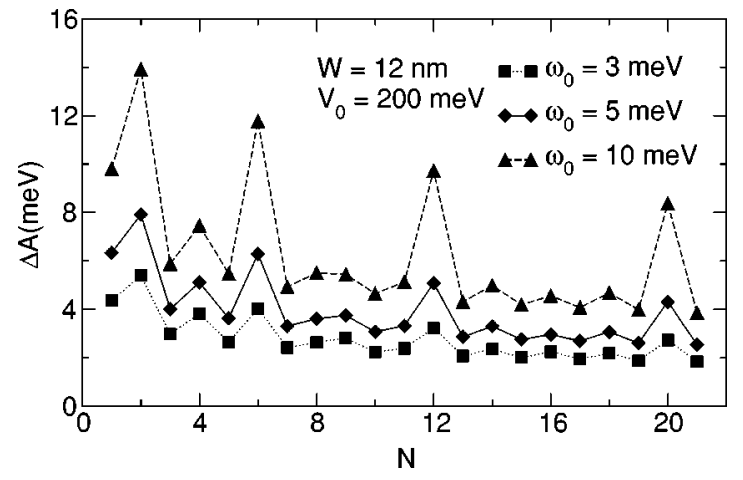

FIG. 1. Addition energies $\Delta A$ as functions of $N$ for a 3D single dot with $V_{0}=200 \mathrm{meV}, W=12 \mathrm{~nm}$, and different $\omega_{0}$ values.

erate electronic states in a shell are filled with parallel spins up to half-shell, in order to maximize the exchange interaction.

Up to $N=12$ the results look very similar to the experimental values, ${ }^{3}$ especially for medium and weak $r$ confinement. For larger $N$ values we have found a conspicuous even-odd effect, instead of the weak maxima experimentally found at $N=20$, and especially at $N=16$. This result does not mean that Hund's rule is violated within this shell. Indeed, we have found spin alignment up to $N=16$, but the associated energy gain is not enough to produce the local maximum at $N=16$. It is worth pointing out that the energies involved in the definition of $\Delta A(N)$ are very large, compared with the second energy difference. For example, for $\omega_{0}=5 \mathrm{meV}$ and $E(16)=1.048 \mathrm{eV}$, whereas $\Delta A(16)$ is $\sim 3$ $\mathrm{meV}$.

For strong $r$ confinement, our results are quite similar to those in Ref. 19, except for medium and small values. This is not surprising, since in this Ref. the $3 \mathrm{D}$ electron-electron energy is treated in first-order perturbation theory, ${ }^{40}$ and thus one should not expect it to hold if the confinement is not strong enough. The two-dimensional $E(N+1)-E(N)$ results at $B=0$ coincide with those in Ref. 41 when $\omega_{0}$ $=5 \mathrm{meV}$.

Our 3D dot is rather strongly confined in the $z$ direction, and the results for $\Delta A(N)$ are similar to those obtained for pure $2 \mathrm{D}$ dots. We have computed the addition energies using the 2D dot model described in detail in Ref. 39; as shown in

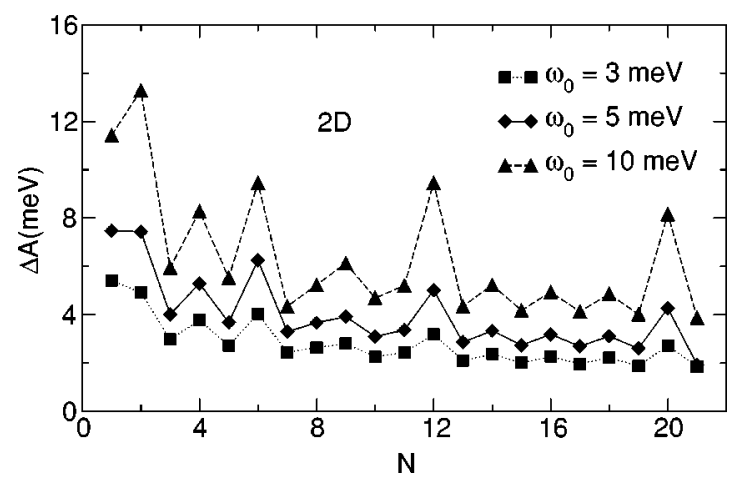

FIG. 2. Addition spectrum as a function of $N$ for a $2 \mathrm{D}$ single dot and different $\omega_{0}$ values.

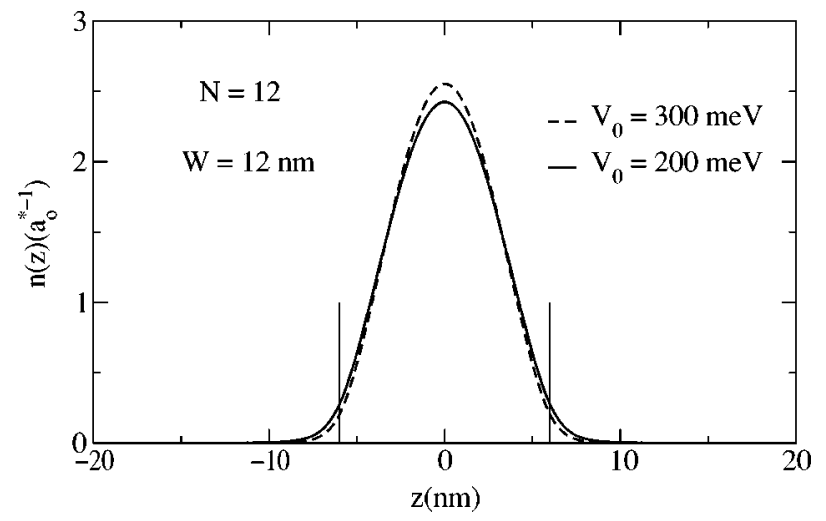

FIG. 3. $n(z)$ densities $\left[\left(a_{0}^{*}\right)^{-1}\right]$ for a 3D single dot with $N$ $=12$, width $W=12 \mathrm{~nm}$, and well depths $V_{0}=200$ and $300 \mathrm{meV}$. The vertical lines indicate the limits of the quantum well.

Fig. 2, 2D and 3D results are very similar, and qualitatively better than those from other different $2 \mathrm{D}$ models. ${ }^{42}$ As one increases the confinement in the $z$ direction up to $V_{0}$ $=300 \mathrm{meV}$, the addition energies become indistinguishable in the scale of Fig. 1. We shall see that this is not the case for double dots, and that even qualitative differences appear if the dots are strongly coupled.

In spite of this quasi-two-dimensional behavior, the electron density spreads beyond the well up to distances that are relevant for vertically coupled dots. This is illustrated in Fig. 3 , where we have plotted the $n(z)$ electron density defined as

$$
n(z) \equiv \int_{0}^{\infty} d r r n(r, z)
$$

corresponding to $N=12, W=12 \mathrm{~nm}$, and $V_{0}=200$ and 300 meV [note that $\left.N=2 \pi \int d z n(z)\right]$. The electron densities spill out of the quantum well, the smaller the confinement, the larger the effect.

Finally, in Fig. 4 we represent the density of a 2D dot hosting 12 electrons, and the $n(r)$ density of the corresponding 3D dot defined as $n(r)=\int d z n(r, z)$ [note that $N$ $\left.=2 \pi \int \operatorname{drrn}(r)\right]$, both for $\omega_{0}=5 \mathrm{meV}$. These radial densities

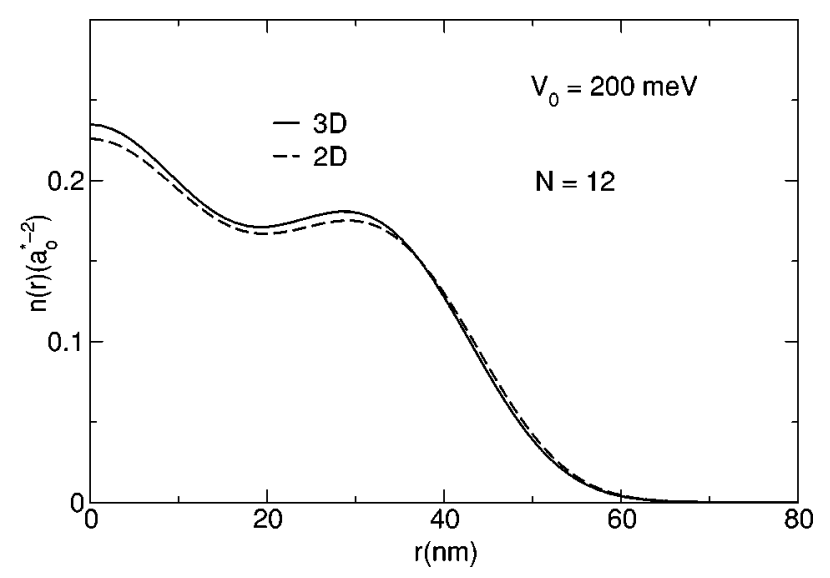

FIG. 4. $n(r)$ densities $\left[\left(a_{0}^{*}\right)^{-2}\right]$ for 2D and 3D single dots with $N=12$ and $\omega_{0}=5 \mathrm{meV}$. The $3 \mathrm{D}$ well width and depth are $W$ $=12 \mathrm{~nm}$ and $V_{0}=200 \mathrm{meV}$, respectively. 


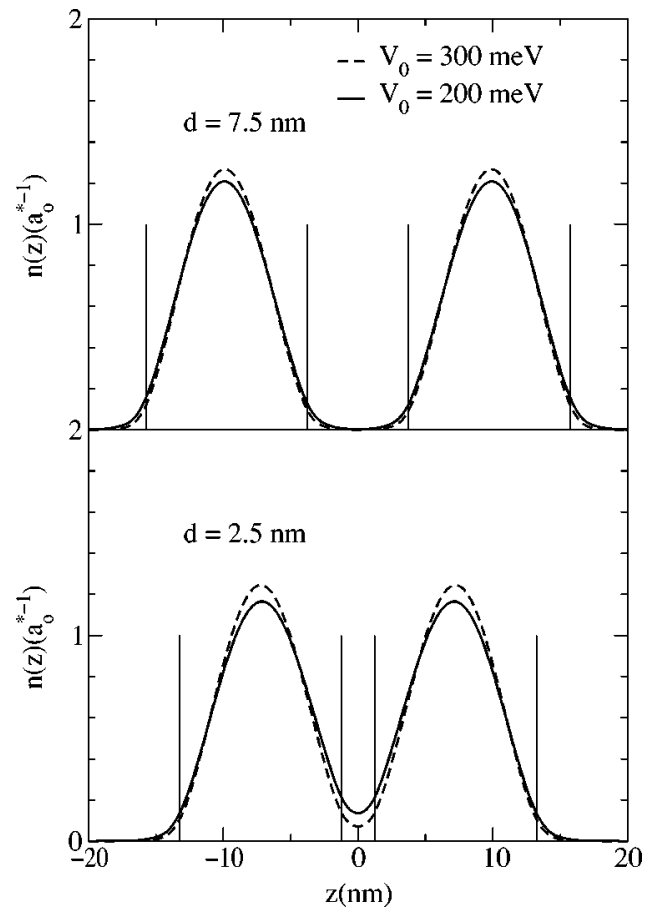

FIG. 5. $n(z)$ densities $\left[\left(a_{0}^{*}\right)^{-1}\right]$ for an $N=12$ double dot of width $W=12 \mathrm{~nm}$, well depth $V_{0}$, and barrier thickness $d$. Top panel, $d=7.5 \mathrm{~nm}$. Bottom panel, $d=2.5 \mathrm{~nm}$. The vertical lines indicate the limits of the double quantum well.

are very similar. The $3 \mathrm{D}$ densities $n(r)$ for $V_{0}=300 \mathrm{meV}$, instead of $200 \mathrm{meV}$, are indistinguishable within the scale of the figure.

These comparisons allow one to infer that the experimental dots are quasi-two-dimensional systems to a large extent, with moderate lateral confinement, $\omega_{0} \leqslant 5 \mathrm{meV}$.

\section{B. Double quantum dots}

We have modeled a symmetric double dot by a parabolic confining potential with frequency $\omega_{0}=5 \mathrm{meV}$ in the $r$ direction, and a symmetric double quantum well in the $z$ direction. Each quantum well has a width equal to $12 \mathrm{~nm}$, and is separated from the other by a barrier of thickness $d$ that varies from 1 to $9 \mathrm{~nm}$. Some experimental results are available $^{4}$ for this system at $d \geqslant 2.5 \mathrm{~nm}$. Results for well depths $V_{0}=200$ and $300 \mathrm{meV}$ will be discussed.

Figure 5 shows the $n(z)$ density profiles corresponding to a quantum-mechanically coupled configuration $(d$ $=2.5 \mathrm{~nm}$ ), and to an electrostatically coupled configuration $(d=7.5 \mathrm{~nm})$. Only in the former case, are the electrons delocalized. A quantitative measure of this localization is provided by the energy splitting between symmetric and antisymmetric sp states (more precisely between even and odd states with respect to specular reflection $z \rightarrow-z$ ), $\Delta_{S A S}$. Indeed, for $N=1$ and large distances, the symmetric and antisymmetric states are degenerate, and $\Delta_{S A S}$ approaches zero. As $d$ decreases, the coupling increases and so does $\Delta_{S A S}$. This effect depends weakly on $N$. We have plotted $\Delta_{S A S}$ as a function of $d$ in Fig. 6, for the lowest $l=0$ sp levels of systems with $N=1$ and 20 .

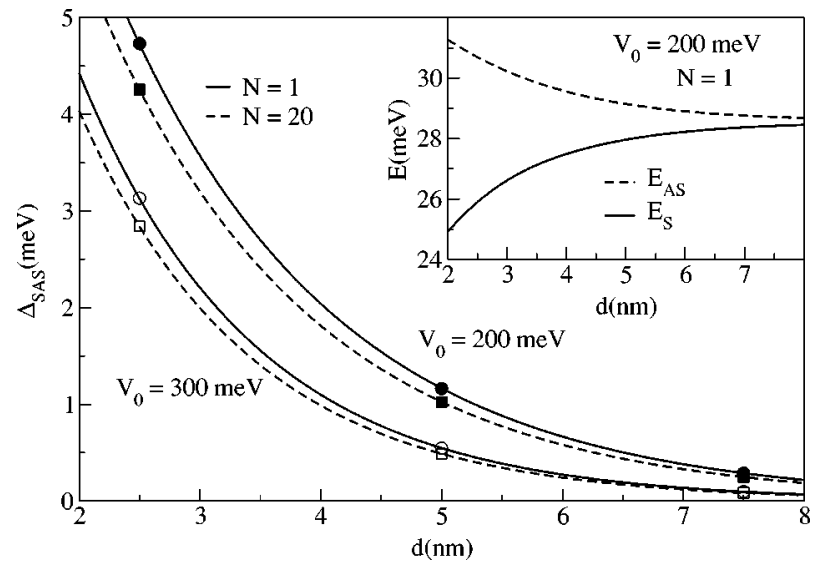

FIG. 6. $\Delta_{S A S}(d)$ for the lowest $l=0$ sp levels of a double dot with $N=1$ and 20. The inset shows the energies $E_{S}$ and $E_{A S}$ defining $\Delta_{S A S}, \Delta_{S A S} \equiv E_{A S}-E_{S}$, for one of the cases presented here.

Due to the characteristics of our double quantum well, $\Delta_{S A S}$ depends exponentially on $d, \Delta_{S A S}(d)=\Delta_{0} \exp (-d /$ $\left.d_{0}\right)$. For $V_{0}=200 \mathrm{meV}$ we have $\left(d_{0}, \Delta_{0}\right)$ $=(1.79 \mathrm{~nm}, 19.2 \mathrm{meV})$ for $N=1$ and $(1.76 \mathrm{~nm}, 17.6 \mathrm{meV})$ for $N=20$, whereas for $V_{0}=300 \mathrm{meV}, \quad\left(d_{0}, \Delta_{0}\right)$ $=(1.44 \mathrm{~nm}, 17.9 \mathrm{meV})$ for $N=1$ and $(1.42 \mathrm{~nm}, 16.5 \mathrm{meV})$ for $N=20$. These values are similar to those in Refs. 4 and 27. It can be seen from Fig. 5 that for a given interdot distance, enlarging $V_{0}$ decreases the coupling between the dots, as the density overlap diminishes. Figure 7 shows the densities $n(r, z)$ for $N=12$ and for two $d$ values corresponding to quantal $(d=2.5 \mathrm{~nm})$ and to electrostatic $(d=7.5 \mathrm{~nm})$ coupling.

Figure 8 shows the addition spectrum for quantummechanically coupled $(d=2.5 \mathrm{~nm})$, and electrostatically coupled $(d=7.5 \mathrm{~nm})$ dots, as well as the results corresponding to the single $3 \mathrm{D}$ dot. They have been obtained for $V_{0}$ $=200 \mathrm{meV}$. Figure 9 shows the same spectrum for $V_{0}$ $=300 \mathrm{meV}$. As expected, changes mostly appear in the strong-coupling case $(d=2.5 \mathrm{~nm})$. Unexpectedly enough, these changes are qualitative, with, for example, maxima in $\Delta A(N)$ changed into minima.

Figure 6 indicates that at $d=7.5 \mathrm{~nm}$ the dots are well apart, only influenced by the electrostatic coupling, and the addition energies are insensitive to the value of $V_{0}$. There are no experimental results in the literature for $\Delta A(N)$ at this interdot distance. However, the experimental analysis ${ }^{4}$ of the derivative of the drain intensity with respect to the drain voltage versus drain voltage indicates that the electrons in the dots are indeed delocalized for $d=2.5 \mathrm{~nm}$, and rather localized for $d=7.5 \mathrm{~nm}$.

The weak coupling at $d=7.5 \mathrm{~nm}$ allows us to interpret the appearance of several peaks in $\Delta A(N)$, such as those at $N$ $=4,8$, and 12 , as due to the fact that two, four, and six electrons on each dot already yield maxima in the addition spectrum of a single QD. The remaining peak at $N=2$ now corresponds to half-filling the first shell in each dot, which would close at $N=2$. This is caused by the localization of one electron on each constituent dot. ${ }^{43}$ 

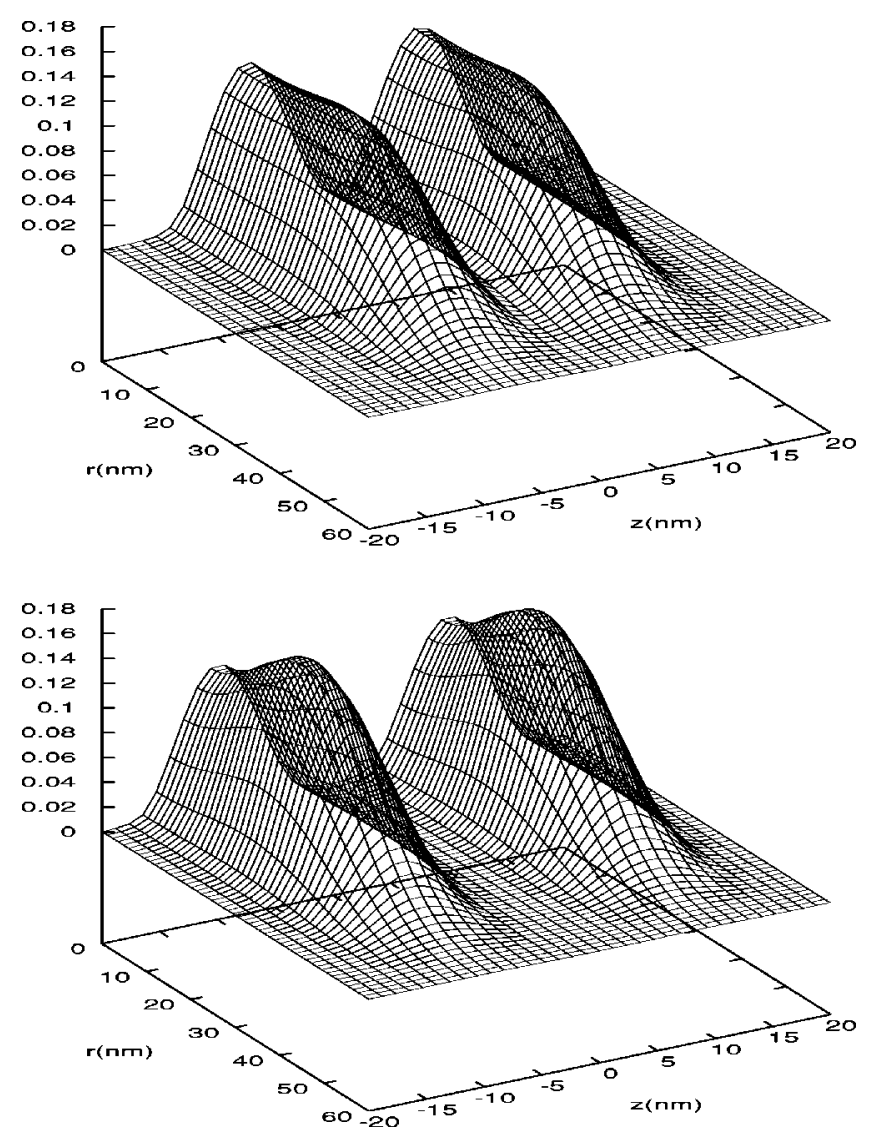

FIG. 7. $n(r, z)$ densities $\left[\left(a_{0}^{*}\right)^{-3}\right]$ for a double dot with $N$ $=12$, width $W=12 \mathrm{~nm}$, well depth $V_{0}=200 \mathrm{meV}$, and barrier thickness $d$. Top panel, $d=2.5 \mathrm{~nm}$. Bottom panel, $d=7.5 \mathrm{~nm}$.

Experimental results have been published ${ }^{4,5}$ for $d$ $=2.5 \mathrm{~nm}$. We have not attempted to use $V_{0}$ as a fitting parameter, so when comparing with experiment one should bear in mind both the sensitivity of $\Delta A(N)$ on the value of $V_{0}$ in the strong coupling limit and the results displayed in Figs. 8 and 9. Published calculations correspond to depths lying in between these two values. ${ }^{22,27}$

One can see that maxima of $\Delta A(N)$ decrease on the average when compared to the isolated QD. This is in agree-

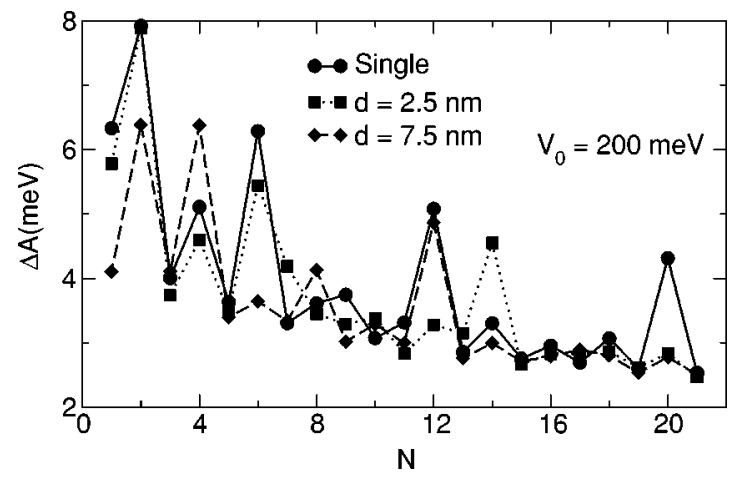

FIG. 8. Addition spectrum as a function of $N$ for a 3D single dot (circles), and for two coupled dots at $d=2.5 \mathrm{~nm}$ (squares) and $d$ $=7.5 \mathrm{~nm}$ (diamonds). The depth of the double quantum well is $V_{0}$ $=200 \mathrm{meV}$.

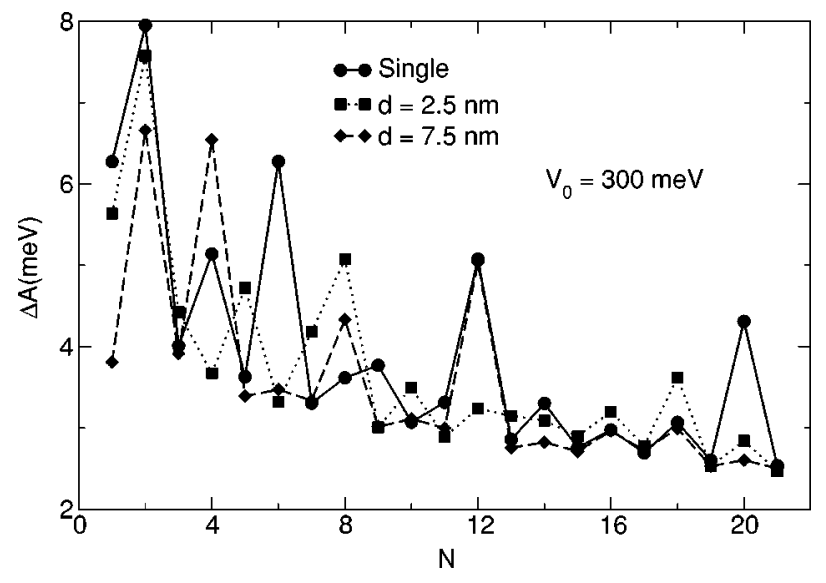

FIG. 9. Same as Fig. 8 for $V_{0}=300 \mathrm{meV}$.

ment with experiment. Note, however, that the experimental $\Delta A(N)$ for the strongly coupled double $\operatorname{dot}^{4}$ is approximately half that corresponding to the single dot, especially when $N \geqslant 10$. This feature is not reproduced by the calculations.

Globally, the results for $d=2.5 \mathrm{~nm}$ are better reproduced with $V_{0}=200 \mathrm{meV}$ up to $N=12$, and with $V_{0}=300 \mathrm{meV}$ for larger $N$ values. Probably, a value of $V_{0}$ in between might improve the agreement with experiment. Other possibilities such as an $N$-dependent $\omega_{0}$ might also be considered, ${ }^{9,27}$ or even some asymmetry in the double well. ${ }^{43}$ We have not tried these possibilities, ${ }^{44}$ but we have checked that selfinteraction corrections (SIC's), which are usually not included in these kind of calculations, do not change the addition spectrum. The results are presented in the Appendix.

For a given electron number, the gs configuration may change as a function of the barrier thickness. The new "phases," i.e., gs configurations which appear as a function of $d$, have been thoroughly discussed. ${ }^{22,27}$ To label them, we have adopted the standard convention of molecular physics for sp electronic orbitals as $\sigma, \pi, \delta, \ldots$, if $l=0,1,2, \ldots$, and upper case Greek letters are used for the total orbital angular momentum. We have also used an adapted version ${ }^{22}$ of ordinary spectroscopic notation ${ }^{2 S+1} L_{g, u}^{ \pm}$where $S$ is the total $\left|S_{z}\right|$, and $L$ is the total $\left|L_{z}\right|$. The superscript $+(-)$ refers to even (odd) states under reflection with respect to the $z=0$ plane, and the subscript $g(u)$ refers to positive (negative) parity states.

We show the evolution with barrier thickness of the energy and gs molecular configuration for several $N$ values in Fig. 10. The vertical lines have been drawn to guide the eye, and different symbols have been used to identify different phases. All panels in the figure display some common trends. Initially, $E(d)$ increases with $d$. The reason for this is twofold. On the one hand, at small $d$ all occupied sp levels are specularly symmetric about the $z=0$ plane (" +" "states), the specularly antisymmetric levels ("-" states) lie at much higher energies (see Fig. 6). On the other hand, the energies of the symmetric and antisymmetric state, respectively, increase and decrease with $d$, and eventually both states become degenerate at large interdot distances. This is a wellknown feature of the one particle, one-dimensional double- 


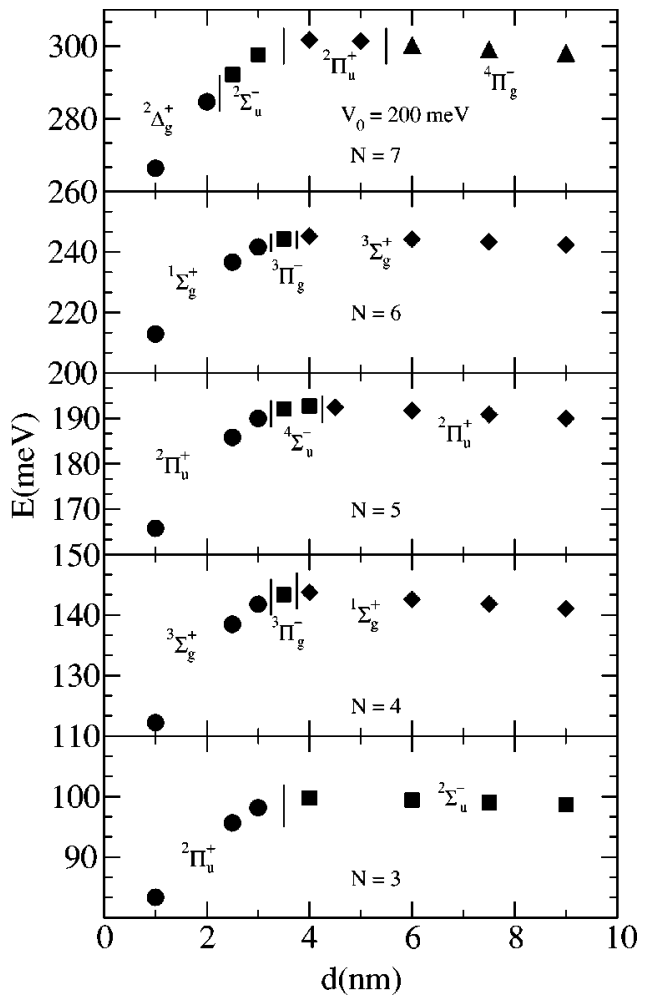

FIG. 10. Energy and ground-state molecular configurations of the double dot as functions of the barrier thickness for $N=3-7$. The depth of the double quantum well is $V_{0}=200 \mathrm{meV}$.

quantum-well problem (see the inset in Fig. 6), which remains valid in the interacting many-electron calculation. The first phase transition takes place when the first antisymmetric sp state becomes occupied. At large distances, $E(d)$ slowly decreases with $d$ due to the decrease of the interdot Coulomb energy. These trends are also present in the Hubbard-like calculations of Ref. 22, but only the lowering of $E(d)$ due to the interdot Coulomb energy is qualitatively reproduced by the calculations of Ref. 27, which fail to yield the energy growth at short distances.

In spite of the difference between the values of the gs energies reported in Refs. 22 and 27, and also with respect to the present work, which has to be mostly attributed to the different confining potentials in each calculation, up to $N$ $=6$ the phases are the same but appear at different $d$ values. In this respect, our results are in closer agreement with those of Ref. 27, possibly because the radial frequencies $\omega_{0}$ are similar in both calculations. We wish to point out that the phase diagram obtained for $V_{0}=300 \mathrm{meV}$ is qualitatively similar to the one shown in Fig. 10, but the phase transitions are shifted $\sim 0.5 \mathrm{~nm}$ to the left.

\section{SUMMARY}

We have used local-spin-density-functional theory to investigate the zero-magnetic-field structure of one single and two identical, vertically coupled QD's of finite thickness. While for one single dot, whose thickness corresponds to that of actual experimental devices, the addition spectrum is quite similar to that predicted by purely two-dimensional

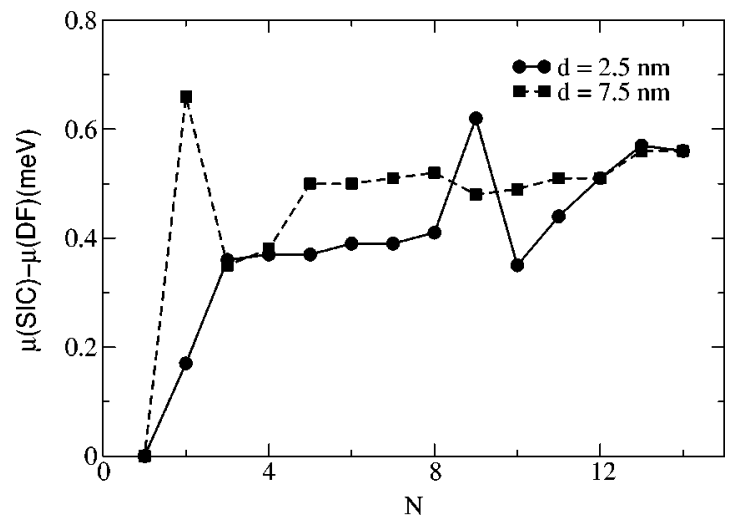

FIG. 11. Difference between SIC and LSDFT addition energies $\mu(N)=E(N)-E(N-1)$ for a double quantum dot of $V_{0}$ $=200 \mathrm{meV}$ depth and barrier thickness $d$ for $N=1-13$.

models, in the case of double dots their vertical extension is essential for a quantitative description of their quantum coupling, which influences the addition spectrum at short distances. For one single dot the calculated addition spectrum compares well with experiment, whereas for two coupled dots the agreement is qualitative. This possibly reflects the fact that in the latter case the spectrum is more sensitive to the actual form of the bare confining potential.

The phase sequence of ground-state configurations which appear as a function of interdot distance is quite similar to that found in previous works, ${ }^{22,27}$ evolving from the "atomic phase" of two strongly coupled dots to the atomic phase of two weakly coupled dots through a series of "moleculartype phases" at intermediate distances. This is a rather robust picture, as it arises from the underlying single-electron structure of the bare confining potential. Indeed, the vertical confinement is so strong that at short distances only symmetric sp states are occupied, the antisymmetric ones lying at quite high energies. This originates the atomic phase of two strongly coupled dots. As the interdot distance increases, the $\Delta_{S A S}$ gap decreases, and the symmetric and antisymmetric sp states eventually become degenerate, originating the atomic phase of two weakly coupled dots. The molecular-type con-

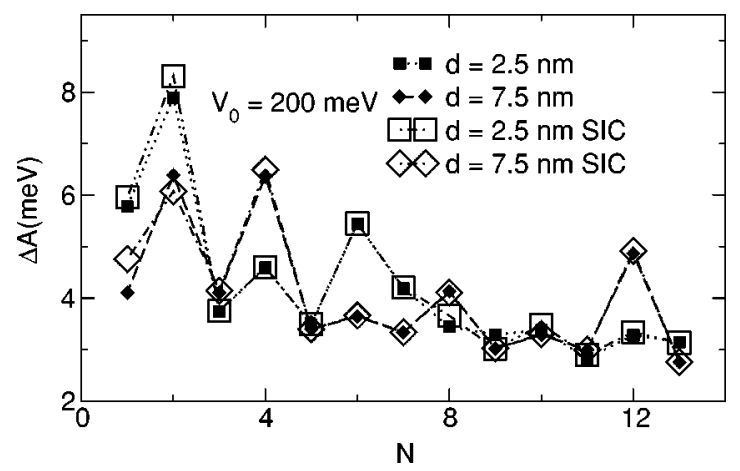

FIG. 12. Addition spectrum as a function of $N$ for two coupled dots at $d=2.5 \mathrm{~nm}$ (squares) and $7.5 \mathrm{~nm}$ (diamonds). The depth of the double quantum well is $V_{0}=200 \mathrm{meV}$. Solid symbols represent LSDFT results, and empty symbols the results obtained after SIC's have been included. 
figurations appear at intermediate distances, where $\Delta_{S A S}$ is similar to the other energy scale of the system, namely $\hbar \omega_{0}$, and when the number of electrons is large enough so that the system can minimize its energy populating antisymmetric states. The larger the number of electrons, the larger the number of populated antisymmetric states. This causes the number of intermediate molecular phases to increase with $N$.

In spite of the mentioned qualitative agreement with previous results, the calculated phase diagrams are as sensitive to the shape of the bare confining potential as the addition spectra. It would be desirable that any prediction of the actual appearance of the phase diagrams should be based on a model that describes, at least qualitatively, the corresponding experimental addition spectrum.

\section{ACKNOWLEDGMENTS}

It is a pleasure to thank Alfredo Poves, Flavio Toigo, and especially Guy Austing for useful discussions. This work was performed under Grant Nos. PB98-1247 and PB98-0124 of the DGESIC, and Grant No. SGR00-0024 of the Generalitat of Catalunya. A.E. acknowledges support from the Dirección General de Enseñanza Superior (Spain).

\section{APPENDIX}

It is well known that the "exact" density functional for the gs energy is self-interaction free (see for instance Ref. 45), but it is not the case of its current approximations, such as the LSDFT. One possible way of removing this drawback is to use the SIC's proposed by Perdew and Zunger, ${ }^{32}$ which introduces an orbital-dependent single-particle potential, that improves the total energy of the electronic system and yields sp eigenvalues which approximate the physical removal energies more closely, at the price of rendering the KS mini- mization far more cumbersome. Since SIC's are relatively more important for few-electron systems, which is the present case, we have tested their effect on the results shown in the body of this paper, in two extreme configurations. We refer the reader to Refs. 32 and 45 for a thorough description of SIC. Here we only give the essential details of the application of the method to our physical problem.

Within the method of Perdew and Zunger, the sp potential in Eq. (1) becomes orbital dependent with the change

$$
\begin{aligned}
V^{H}+V^{x c}+W^{x c} \eta_{\sigma} \rightarrow & V_{\mathrm{eff}} \equiv V^{H}+V^{x c}+W^{x c} \eta_{\sigma}-V^{H}\left[n_{n l \sigma}\right] \\
& -V^{x c}\left[n_{n l \sigma}, n_{n l \sigma}\right]-W^{x c}\left[n_{n l \sigma}, n_{n l \sigma}\right] \eta_{\sigma},
\end{aligned}
$$

where $n_{n l \sigma}=\left|u_{n l \sigma}(r, z)\right|^{2}$ is the "orbital density" and $V^{H}\left[n_{n l \sigma}\right]$ is obtained solving the Poisson equation $\Delta V^{H}\left[n_{n l \sigma}\right]=-4 \pi n_{n l \sigma}(r, z)$ as indicated in Sec. II. After self-consistency is achieved, the total energy can be obtained from Eq. (8), which has been written as

$$
\begin{aligned}
E \rightarrow & E-\frac{1}{2} \sum_{n l \sigma} \int d \mathbf{r} V^{H}\left[n_{n l \sigma}\right] n_{n l \sigma}(\mathbf{r}) \\
& -\sum_{n l \sigma} \int d \mathbf{r} \mathcal{E}_{x c}\left[n_{n l \sigma}, n_{n l \sigma}\right] .
\end{aligned}
$$

In Fig. 11 we show the difference between SIC and LSDFT addition energies defined as $\mu(N)=E(N)-E(N$ -1) corresponding to the $V_{0}=200 \mathrm{meV}$ double quantum dot. The average difference is small, of the order of $0.4-0.5$ $\mathrm{meV}$. This difference almost cancels out in the addition spectrum $\Delta A(N)$, as can be seen in Fig. 12. This constitutes an interesting result in itself, indicating that if one only wishes to obtain the addition spectrum, LSDFT does not need to be corrected for self-interaction effects.
${ }^{1}$ L. Jacak, P. Hawrylak, and A. Wójs, Quantum Dots (Springer, Berlin, 1998).

${ }^{2}$ Mesoscopic Physics and Electronics, edited by T. Ando, Y. Arakawa, K. Furuta, S. Komiyama, and H. Nakashima (Springer, Berlin, 1998).

${ }^{3}$ S. Tarucha, D. G. Austing, T. Honda, R. J. van der Hage, and L. P. Kouvenhoven, Phys. Rev. Lett. 77, 3613 (1996).

${ }^{4}$ D. G. Austing, T. Honda, Y. Tokura, and S. Tarucha, Physica B 249-251, 206 (1998).

${ }^{5}$ S. Tarucha, T. Honda, D. G. Austing, Y. Tokura, K. Muraki, T. H. Oosterkamp, J. W. Janssen, and L. P. Kouwenhoven, Physica E 3, 112 (1998).

${ }^{6}$ S. Sasaki, D. G. Austing, and S. Tarucha, Physica B 256-258, 157 (1998).

${ }^{7}$ T. H. Oosterkamp, J. W. Janssen, L. P. Kouwenhoven, D. G. Austing, T. Honda, and S. Tarucha, Phys. Rev. Lett. 82, 2931 (1999)

${ }^{8}$ D. G. Austing, S. Sasaki, S. Tarucha, S. M. Reimann, M. Koskinen, and M. Manninen, Phys. Rev. B 60, 11514 (1999).

${ }^{9}$ S. M. Reimann, M. Koskinen, M. Manninen, and B. R. Mottel- son, Phys. Rev. Lett. 83, 3270 (1999).

${ }^{10}$ M. Koskinen, S. M. Reimann, and M. Manninen, Phys. Rev. Lett. 79, 1389 (1997).

${ }^{11}$ C. Yannouleas and U. Landman, Phys. Rev. Lett. 82, 5325 (1999); 85, 2220(E) (2000).

${ }^{12}$ A. Puente and Ll. Serra, Phys. Rev. Lett. 83, 3266 (1999).

${ }^{13}$ A. Kumar, S. E. Laux, and F. Stern, Phys. Rev. B 42, 5166 (1990).

${ }^{14}$ A. Natori and D. Nakamura, Jpn. J. Appl. Phys. 38, 380 (1999).

${ }^{15}$ M. Stopa, Phys. Rev. B 54, 13767 (1996).

${ }^{16} \mathrm{~S}$. Nagaraja, Ph. Matagne, V.-Y. Thean, J.-P. Leburton, Y.-H. Kim, and R. M. Martin, Phys. Rev. B 56, 15752 (1997).

${ }^{17}$ I.-H. Lee, V. Rao, R. M. Martin, and J.-P. Leburton, Phys. Rev. B 57, 9035 (1998).

${ }^{18}$ I.-H. Lee, K.-H. Ahn, Y.-H. Kim, R. M. Martin, and J.-P. Leburton, Phys. Rev. B 60, 13720 (1999).

${ }^{19}$ M. Rontani, F. Rossi, F. Manghi, and E. Molinari, Appl. Phys. Lett. 72, 957 (1998).

${ }^{20}$ M. Rontani, F. Rossi, F. Manghi, and E. Molinari, Phys. Rev. B 59, 10165 (1999). 
${ }^{21}$ T. Schmidt, R. J. Haug, K. v. Klitzing, A. Förster, and H. Lüth, Phys. Rev. Lett. 78, 1544 (1997).

${ }^{22}$ M. Rontani, F. Rossi, F. Manghi, and E. Molinari, Solid State Commun. 112, 151 (1999).

${ }^{23}$ J. J. Palacios and P. Hawrylak, Phys. Rev. B 51, 1769 (1995).

${ }^{24}$ O. Mayrock, S. A. Mikhailov, T. Darnhofer, and U. Rössler, Phys. Rev. B 56, 15760 (1997).

${ }^{25}$ B. Partoens, V. A. Schweigert, and F. M. Peeters, Phys. Rev. Lett. 79, 3990 (1997).

${ }^{26}$ B. Partoens, A. Matulis, and F. M. Peeters, Phys. Rev. B 57, 13039 (1998).

${ }^{27}$ B. Partoens and F. M. Peeters, Phys. Rev. Lett. 84, 4433 (2000).

${ }^{28}$ K. T. Davies, H. Flocard, S. Krieger, and M. S. Weiss, Nucl. Phys. A 342, 112 (1980).

${ }^{29}$ M. Weissbluth, Atoms and Molecules (Academic Press, New York, 1978).

${ }^{30}$ M. Barranco, J. Navarro, and A. Poves, Phys. Rev. Lett. 78, 4729 (1997).

${ }^{31}$ K. Hirose and N. S. Wingreen, Phys. Rev. B 59, 4604 (1999).

${ }^{32}$ J. P. Perdew and A. Zunger, Phys. Rev. B 23, 5048 (1981).

${ }^{33}$ S. Lundqvist, Theory of the Inhomogeneous Electron Gas, edited by S. Lundqvist and N. H. March (Plenum, New York, 1983), p. 149.

${ }^{34}$ D. M. Ceperley and B. J. Alder, Phys. Rev. Lett. 45, 566 (1980).
${ }^{35}$ S. H. Vosko, L. Wilk, and M. Nusair, Can. J. Phys. 58, 1200 (1980).

${ }^{36}$ M. Abramowitz and I. A. Stegun, Handbook of Mathematical Functions (Dover, New York, 1970).

${ }^{37}$ W. H. Press, S. A. Teukolsky, W. T. Vetterling, and B. P. Flannery, Numerical Recipes (Cambridge University Press, Cambridge, 1992).

${ }^{38}$ For the sake of comparison of, the $r$ step we have routinely used (Ref. 39) in 2D circularly symmetric calculations is $0.06 a_{0}^{*}$.

${ }^{39}$ M. Pi, M. Barranco, A. Emperador, E. Lipparini, and Ll. Serra, Phys. Rev. B 57, 14783 (1998).

${ }^{40}$ E. Merzbacher, Quantum Mechanics (Wiley, New York, 1970).

${ }^{41}$ O. Steffens, U. Rössler, and M. Suhrke, Europhys. Lett. 42, 529 (1998).

${ }^{42}$ M. Macucci, K. Hess, and G. J. Iafratt, Phys. Rev. B 55, R4879 (1997).

${ }^{43}$ Y. Tokura, D. G. Austing, and S. Tarucha, J. Phys.: Condens. Matter 11, 6023 (1999).

${ }^{44}$ We have checked, however, that the $\omega_{0}(N)$ laws proposed in Refs. 9 and 27, applied to one circularly symmetric 2D quantum dot at $B=0$ yield an addition spectrum, in rather poor agreement with the experimental spectrum (Ref. 3).

${ }^{45}$ R. M. Dreizler and E. K. U. Gross, Density Functional Theory (Springer-Verlag, Berlin, 1990). 